\title{
Mean flow produced by small-amplitude vibrations of a liquid bridge with its free surface covered with an insoluble surfactant
}

\author{
Luis M. Carrión \\ Universidad de las Fuerzas Armadas-ESPE, Av. Gral. Rumiahui s/n, Sangolqu 171103, Ecuador \\ Miguel A. Herrada \\ Escuela Técnica Superior de Ingenieros, Universidad de Sevilla, Avda. de los Descubrimientos s/n, E-41092-Sevilla, Spain \\ José M. Montanero \\ Departmento de Ingeniería Mecánica, Energética y de los Materiales and Instituto de Computación Científica Avanzada (ICCAEx), \\ Universidad de Extremadura, Avda. de Elvas s/n, E-06071 Badajoz, Spain \\ José M. Vega \\ E.T.S.I. Aeronáutica y del Espacio, Universidad Politécnica de Madrid, Plaza Cardenal Cisneros 3, 28040 Madrid, Spain
}

(Received 28 April 2017; published 1 September 2017)

\begin{abstract}
As is well known, confined fluid systems subject to forced vibrations produce mean flows, called in this context streaming flows. These mean flows promote an overall mass transport in the fluid that has consequences in the transport of passive scalars and surfactants, when these are present in a fluid interface. Such transport causes surfactant concentration inhomogeneities that are to be counterbalanced by Marangoni elasticity. Therefore, the interaction of streaming flows and Marangoni convection is expected to produce new flow structures that are different from those resulting when only one of these effects is present. The present paper focuses on this interaction using the liquid bridge geometry as a paradigmatic system for the analysis. Such analysis is based on an appropriate post-processing of the results obtained via direct numerical simulation of the system for moderately small viscosity, a condition consistent with typical experiments of vibrated millimetric liquid bridges. It is seen that the flow patterns show a nonmonotone behavior as the Marangoni number is increased. In addition, the strength of the mean flow at the free surface exhibits two well-defined regimes as the forcing amplitude increases. These regimes show fairly universal power-law behaviors.
\end{abstract}

DOI: 10.1103/PhysRevE.96.033101

\section{INTRODUCTION}

Oscillatory flows in confined and low-viscosity fluid systems develop oscillatory boundary layers near solid walls and interfaces. Reynolds stresses exhibit nonzero temporal mean values in these layers, where they drive a mean flow. This interesting effect was first shown in a pioneering work by Lord Rayleigh [1] when explaining the anomalous sand accumulation at the bottom of a vertically vibrated container, observed 50 years earlier by Faraday [2]. Rayleigh also noted that the same mechanism was seemingly responsible for nonuniform dust accumulation (known as Kundt figures) at the walls of sound tubes [3] and for the formation of steady vortical structures in vibrated soap films, already observed by Sedley Taylor [4]. This latter problem has been more recently studied by Vega et al. [5]. They concluded that, under reasonable assumptions and according to Rayleigh's insight, the observed vortical structures are most seemingly due to the streaming flow in the air surrounding the soap film. Rayleigh made a careful analysis of the (nowadays called) boundary layer attached to a (no-slip) solid boundary, obtaining the steady mean flow responsible for sand or dust nonuniform accumulation near the boundary. Rayleigh's work was completed 50 years later by Schlichting [6] (see also Ref. [7]), who noticed that the streaming flow velocity was nonzero at the internal edge of the boundary layer, which is a driving mechanism for the generation of mean flows in the fluid bulk. Longuet-Higgins [8] made a similar analysis for the streaming flow within the boundary layer attached to a free surface, which again was nonzero at the internal edge of the layer and thus also responsible for the generation of a global mean flow in the bulk. An important well-known property is that the time-averaged velocity or stress converges to a generally nonzero value as viscosity goes to zero, while it would vanish if viscosity were identically zero (i.e., when the boundary layer is absent) [9]. This seemingly surprising effect is explained by the singular perturbation character of the inviscid limit.

More recently, the mean flow (also called steady or acoustic streaming $[10,11]$ ) has been studied in various contexts. In particular, the streaming flow induced by no-slip boundaries has been studied in connection with flows in blood vessels [12], generation of mean motions in the ear [13], interaction of sound waves with bodies [14], flows around vibrating bodies [15], and streaming-flow-jets produced by acoustic waves [16]. On the other hand, the streaming flow produced in the boundary layer attached to a vibrating free surface has an effect on the dynamics of oscillating bubbles [17], is of interest in water wave theory [18-22], and has been shown to play a role in the instability of the ocean Langmuir circulations [23]. In different contexts, the streaming flow has been studied in microfluidic systems [24] and in conjunction with thermal effects for various purposes, including the control of thermocapillary convection at low viscosity [25-28] and cooling in narrow channels [29].

For nearly resonant systems at small viscosity, the streaming flow is not just a byproduct of the primary waves, but 
generally affects the dynamics of those waves, as repeatedly shown for various flow configurations and vibrating devices [30-34]. This is because the streaming flow velocity scales with the square of the wave amplitude and produces an effect in the amplitude equations governing the dynamics of the primary waves that is proportional to the product of the primary waves amplitude and the streaming flow velocity. Thus, this latter effect scales as the cube of the primary waves amplitude, which is the order of magnitude of the cubic nonlinearity that saturates the dynamics in these systems [35].

The streaming flow is responsible for the effective mass transport in the fluid system and, in particular, for the overall motion of passive scalars and surfactants. Therefore, the mean flow is expected to exhibit a nontrivial interaction with Marangoni convection. The understanding of this interaction has been pursued in a number of papers. For instance, Martin and Vega [36] included the effect of Marangoni elasticity in a previous theory for clean free surfaces [32] to study the drift stability of standing Faraday waves in annular containers, previously found experimentally by Douady et al. [37]. More recently, Strickland et al. [38] have pointed out that materials (such as crude oil, biogenic slicks, or industrial and medical surfactants) absorbed at the fluid free surface are expected to move in response to surface waves. They have experimentally studied such effect for Faraday waves in a shallow cylindrical container with an insoluble surfactant monolayer. They distinguished the effects of the meniscus waves near the boundaries and the travelling waves in the far field, which resulted in very complex spatiotemporal patterns. The case of Faraday waves in a rectangular container was considered in Ref. [39], where no specific surfactant was added. In this study, surface contamination was seemingly present due to the use of tap water, which is easily contaminated. In fact, water contamination was also most probably responsible for some striking mean flow effects due to wave-maker oscillations that have been recently reported [40].

The streaming flow theory and systems mentioned above all deal with the low-viscosity limit, in which the thickness of the oscillatory boundary layers are very small compared to the characteristic lengths of the primary surface waves. This sets an upper limit to the surface waves oscillation frequency. If that condition does not hold, the streaming flow can still be analyzed by appropriate time-averaging of the trajectories of fluid elements calculated via direct numerical simulation. This task has not been addressed in the literature, to our knowledge.

As mentioned above, Faraday waves in vibrating containers may produce meniscus oscillations that further complicate the dynamics, and may mask the interaction between the streaming flow and Marangoni convection. To overcome this difficulty, we consider a vibrating liquid bridge with the contact lines attached to the edges of the supporting disks, as we have done in other related works dealing with surfactant monolayer dynamics $[41,42]$. This geometry has the additional advantage that direct numerical simulation is feasible at a reasonable computational price. The liquid bridge geometry has already been considered in connection with the streaming flow produced by vibrations, focusing on weakly nonlinear oscillations [43] and the effect of free surface waves [44]. However, these studies dealt with the low viscosity limit. On the contrary, viscosity is not necessarily small in the present paper. Our analysis is based on direct numerical simulation for arbitrary values of that parameter.

Concerning the interaction of the streaming flow with Marangoni convection, we may anticipate here that there is a tradeoff between both. Without Marangoni elasticity (the surfactant is treated as a passive scalar), the surfactant is transported by the streaming flow, which gives rise to nonuniform passive scalar concentrations at the free surface. However, Marangoni elasticity reacts against surfactant concentration gradients, decreasing the tangential velocity at the free surface and counterbalancing the effect of the streaming flow alone. Thus, the resulting streaming flow patterns are expected to be highly affected by Marangoni stresses, at least near the free surface.

The main object of this paper is to analyze the interaction between the streaming flow and Marangoni elasticity in a vibrated liquid bridge using direct numerical simulation. For simplicity, we consider an insoluble surfactant monolayer on top of the interface of a vibrating liquid bridge. We ignore gravity, consider the symmetric case in which the disks supporting the bridge have equal radii, and neglect surface viscosities. The streaming flow velocity and the time average of both the liquid bridge shape and the surfactant distribution will be calculated in two ways, one asymptotic assuming that the oscillatory flow intensity goes to zero, and another "exact" that does not take that assumption into account. In the former case, the streaming flow velocity is calculated as the sum of the mean Eulerian velocity and the Stokes drift, while in the latter case that velocity is obtained through the time-averaging of the trajectories of the fluid elements. These two approaches rely on the appropriate post-processing of the results obtained by direct numerical simulation, and their outcomes will be used for cross-checking.

The remainder of the paper is organized as follows. The theoretical background is considered in Sec. II. This section includes the problem formulation and a description of the numerical solver in Sec. II A, and the derivation of the equations giving the streaming flow velocity and the time average of both the liquid bridge shape and the surfactant distribution in Sec. II B. The main results of the paper are presented in Sec. III, considering separately the influence of the Marangoni elasticity and the intensity of the oscillatory flow in Sec. III A and Sec. III B, respectively. The paper ends with some concluding remarks in Sec. IV.

\section{THEORETICAL BACKGROUND}

As anticipated, the analysis of the interaction between the streaming flow and Marangoni stress will be conducted by post-processing "exact results" obtained upon direct numerical simulation. The theoretical background in Sec. II A includes the problem formulation, the description of the numerical solver, and the nondimensional parameter range considered. The post-processing formulas to compute the streaming flow is derived and discussed in Sec. II B.

\section{A. Governing equations and numerical solver}

We consider a liquid bridge (sketched in Fig. 1) of length $L$ and volume $\mathcal{V}$, held between two circular disks of equal radius 


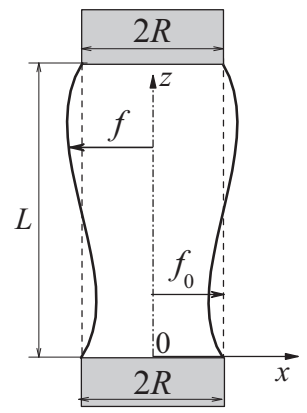

FIG. 1. Liquid bridge configuration.

$R$, and surrounded by another fluid medium of negligible density and viscosity. Nondimensionalization of the governing equations is made by using $R$ and $t_{0}=\left(\rho R^{3} / \sigma_{0}\right)^{1 / 2}$ as units for length and time, respectively, where $\rho$ is the liquid density and $\sigma_{0}$ is the equilibrium (initial) surface tension. For axisymmetric flows, the incompressible Navier-Stokes equations are

$$
\begin{gathered}
(r u)_{r}+r w_{z}=0, \\
u_{t}+u u_{r}+w u_{z}=-p_{r}+C\left[u_{r r}+(u / r)_{r}+u_{z z}\right], \\
w_{t}+u w_{r}+w w_{z}=-p_{z}+C\left[w_{r r}+w_{r} / r+w_{z z}\right],
\end{gathered}
$$

where $r$ and $z$ are the radial and axial cylindrical coordinates, respectively, $t$ is the time variable, the subscripts $r, z$, and $t$ denote hereinafter partial derivatives with respect to those variables, $u$ and $w$ are the radial and axial velocity components, respectively, $p$ is the (hydrostatic) reduced pressure, and $C=$ $\mu\left(\rho \sigma_{0} R\right)^{-1 / 2}$ is the volumetric Ohnesorge number defined in terms of the bulk viscosity $\mu$.

As boundary conditions, we consider the regularity conditions $u=w_{r}=p_{r}=0$ at the symmetry axis $r=0$, as well as the kinematic compatibility condition

$$
f_{t}+f_{z} w-u=0,
$$

and the equilibrium of normal and tangential stresses,

$$
\begin{gathered}
p-B(t) z-\widehat{\sigma} \kappa-\frac{2 C\left[u_{r}-f_{z}\left(w_{r}+u_{z}\right)+f_{z}^{2} w_{z}\right]}{1+f_{z}^{2}}=0, \\
C \frac{\left[1-f_{z}^{2}\right]\left(w_{r}+u_{z}\right)+2 f_{z}\left(u_{r}-w_{z}\right)}{1+f_{z}^{2}}=\frac{\widehat{\sigma}_{z}}{\sqrt{1+f_{z}^{2}}},
\end{gathered}
$$

at the free surface, $r=f(z, t)$. It must be noted that these boundary conditions do not apply if overturning takes place, which may occur just before the pinch-off for vibration amplitudes larger than those considered in this paper. In the above equations,

$$
\kappa=\frac{1+f_{z}^{2}-f f_{z z}}{f\left(1+f_{z}^{2}\right)^{3 / 2}}
$$

is (twice) the mean curvature of the free surface, $B(t)$ is the unsteady Bond number defined in Eq. (12), and $\widehat{\sigma}=\sigma / \sigma_{0}$ is the ratio of the local surface tension value $\sigma$ to its equilibrium value $\sigma_{0}$. The dependence of the surface tension on the surfactant concentration obeys different relationships, depending on the surfactant. Here, we shall use the Szyskowski-Frumkin equation of state [45],

$$
\widehat{\sigma}=1+\operatorname{Ma} \ln \left(\frac{1-\widehat{\gamma}}{1-\widehat{\gamma_{0}}}\right),
$$

where $\widehat{\gamma}=\gamma / \gamma_{\infty}$ and $\widehat{\gamma}_{0}=\gamma_{0} / \gamma_{\infty}$ are the local surface coverage and the equilibrium surface coverage, respectively. These quantities are defined in terms of the local surfactant surface concentration $\gamma$, its equilibrium value $\gamma_{0}$, and its maximum value $\gamma_{\infty}$ corresponding to the maximum surfactant packing density. In addition, $\mathrm{Ma}=\gamma_{\infty} R_{g} T_{e} / \sigma_{0}$ is the (Marangoni) elasticity number defined in terms of $\gamma_{\infty}$, the gas constant $R_{g}$, the temperature $T_{e}$, and the equilibrium surface tension $\sigma_{0}$. On the other hand, the surfactant concentration along the free surface must obey the surfactant conservation equation [42],

$$
\begin{gathered}
{\left[f\left(1+f_{z}^{2}\right)^{1 / 2} \widehat{\gamma}\right]_{t}+\left[f\left(1+f_{z}^{2}\right)^{1 / 2} w \widehat{\gamma}\right]_{z}} \\
=\left[\frac{f}{\operatorname{Pe}^{S}} \frac{\widehat{\gamma} z}{\sqrt{1+f_{z}^{2}}}\right]_{z} \text { at } \quad r=f(z, t),
\end{gathered}
$$

where $\mathrm{Pe}^{S}=R^{2} /\left(t_{0} D^{S}\right)$ is the surface Peclet number, and $D^{S}$ is the surface diffusion coefficient.

The boundary condition Eq. (5) and the conservation Eq. (9) must be complemented with the anchoring condition for the free surface and impenetrability of the surfactant at the liquid bridge disks,

$$
f=1 \text { and } \hat{\gamma}_{z}=0 \text { at } z=0 \text { and } 2 \Lambda,
$$

where $\Lambda=L /(2 R)$ is the slenderness. Also, the nondimensional volume $V \equiv \mathcal{V} /\left(\pi R^{2} L\right)$ and the total amount of surfactant are both conserved, namely,

$$
\begin{aligned}
\int_{0}^{2 \Lambda} f^{2} d z & =\int_{0}^{2 \Lambda} f_{0}^{2} d z \equiv 2 \Lambda V, \quad \int_{0}^{2 \Lambda} \sqrt{1+f_{z}^{2}} f \hat{\gamma} d z \\
& =\int_{0}^{2 \Lambda} \sqrt{1+f_{0 z}^{2}} f_{0} \hat{\gamma}_{0} d z
\end{aligned}
$$

where $f_{0}$ is the initial value of the free surface position.

The initial liquid bridge shape is calculated by integrating the Young-Laplace equation, and the initial surfactant concentration is set as spatially constant. For $t \geqslant 0$, a rigid-solid acceleration with magnitude $a \omega^{2} e^{i \omega t}+$ c.c. is applied, where $a$ and $\omega$ are the forcing amplitude and frequency, respectively, $i$ is the imaginary unit, and c.c. stands hereinafter for the complex conjugate. Therefore, the unsteady Bond number becomes

$$
B(t)=\hat{B} e^{i \omega t}+\text { c.c. },
$$

where $\hat{B}=\rho a \omega^{2} R^{2} / \sigma_{0}$ is the dynamical Bond number. The above theoretical model is formulated in terms of the eight nondimensional parameters:

$$
\Lambda, \quad V, \quad C, \mathrm{Pe}^{S}, \mathrm{Ma}, \widehat{\gamma}_{0}, \omega, \text { and } \hat{B} .
$$

We have selected the values of those parameters according to the following considerations:

(i) Because of the large dimension of the parameter space, the analysis will be restricted to (equilibrium) cylindrical shapes $(V=1)$ with $\Lambda=1.25$. 
(ii) The volumetric Ohnesorge number will be selected as $C=0.01$, which corresponds to a typical experimental realization with millimetric water liquid bridges. To analyze the influence of the liquid viscosity, the volumetric Ohnesorge number $C=0.001$ will be considered too.

(iii) The surfactant surface diffusivity is typically of the order of $10^{-10}-10^{-9} \mathrm{~mm}^{2} / \mathrm{s}$ [45], which leads to surface Peclet numbers in the range $10^{5}-10^{6}$ when those surfactants are added to millimetric liquid bridges. Thus, the influence of surface diffusion on the liquid bridge dynamics can be ignored. However, ignoring surface diffusion makes the surfactant conservation Eq. (9) (hyperbolic and) quite stiff. Because the physics of the high-Peclet-number limit is well captured using much smaller values of $\mathrm{Pe}^{S}$, we will consider $\mathrm{Pe}^{S}=1000$ in the numerical simulations.

(iv) The elasticity number $\mathrm{Ma}=1$ corresponds to a strong surfactant. For this reason, we will consider the interval $0 \leqslant$ $\mathrm{Ma} \leqslant 1$

(v) The formation of micelles for $\widehat{\gamma} \gtrsim 1$ may add rheological effects not considered in this paper. For this reason, the value $\widehat{\gamma_{0}}=0.5$ will be selected as typical for the surfactant concentration.

(vi) The main goal of this paper is the analysis of the influence of the Marangoni convection on the streaming flow caused by the liquid bridge oscillation modes. However, both the thickness of the boundary layers and the axial characteristic length decrease as the oscillation frequency increases, which leads to a considerable increase of the spatial resolution required in the simulations. For this reason, all the calculations will be made for forcing frequencies around the first resonance frequency of the liquid bridge. The value of this quantity is about 2.1 for the equilibrium shape considered in the first item.

(vii) The strength of the oscillation must be not too large for the quasi-linear theory developed in this paper to be valid. In particular, the steepness of the free surface deflection must be sufficiently small. Taking into account these considerations, $0<\hat{B}<0.05$ is an appropriate range for the dynamical Bond number.

Summarizing the above, the following values of the nondimensional parameters listed in Eq. (13) will be considered in most of the paper:

$$
\begin{aligned}
\Lambda & =1.25, \quad V=1, \quad C=0.01 \text { or } 0.001, \\
\operatorname{Pe}^{S} & =1000, \quad 0 \leqslant \mathrm{Ma} \leqslant 1, \\
\widehat{\gamma_{0}} & =0.5, \quad 2 \leqslant \omega \leqslant 2.2, \quad \text { and } 0<\hat{B}<0.05 .
\end{aligned}
$$

The problem formulated above will be solved with the numerical method recently proposed in Ref. [46]. The timedependent fluid domain is mapped onto a fixed rectangular domain $(0 \leqslant \eta \leqslant 1,0 \leqslant z \leqslant 2 \Lambda)$ through the radial coordinate transformation $\eta=r / f(z, t)$. The numerical domain is discretized (after some calibration) using $n_{\eta}=41$ and $n_{z}=101$ grid points along the $\eta$ and $z$ directions, respectively. The spatial derivatives are calculated with fourth-order finite differences. The (implicit) time advancement is performed using second-order backward-differences with a fixed time step $\Delta t=T / 80$, where $T=2 \pi / \omega$ is the period of the forced

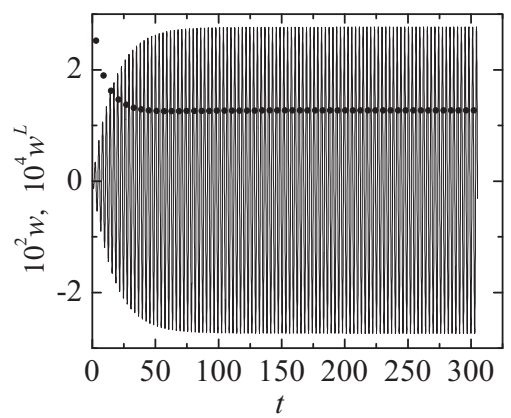

FIG. 2. Time evolution of the axial component $w$ of the velocity numerically calculated (solid line) and of the axial component $w^{L}$ of the Lagrangian mean velocity (dots). These quantities were evaluated at the liquid bridge position $(r=1, z=3 \Lambda / 2)$. The component $w^{L}$ was obtained from the difference between the fluid element positions at the instants $t$ and $t-T$ [Eq. (24)]. The simulation was conducted for $\Lambda=1.25, V=1, C=0.01, \mathrm{Ma}=0, \omega=2.1$, and $\hat{B}=0.005$.

oscillation. At each time step, the resulting set of algebraic equations are solved with the iterative Newton-Raphson technique. The elements of the Jacobian of the discretized system of equations are computed via standard symbolic software at the outset, before running the simulation. These functions are evaluated numerically in the Newton-Raphson iterations to find the solution at each time step, which reduces considerably the required CPU time. The initial guess for the iterations at each time step is the solution at the previous instant.

\section{B. Streaming flow}

Under the conditions mentioned above, we assume that the oscillatory flow velocity and free surface deflection are both conveniently small. Thus, after a transient stage (see Fig. 2), this flow is assumed to be quasilinear, periodic, and quasimonochromatic with the forcing frequency $\omega$. To the approximation relevant in this paper, the velocity components, the free surface deflection, and the surfactant concentration can be written as

$$
\begin{aligned}
(u, w)= & \varepsilon\left[(U(r, z), W(r, z)) e^{i \omega t}+\text { c.c. }\right] \\
& +\varepsilon^{2}\left[\left(U_{2}(r, z), W_{2}(r, z)\right) e^{2 i \omega t}+\right.\text { c.c. } \\
& \left.+\left(u^{m}(r, z), w^{m}(r, z)\right)\right]+O\left(\varepsilon^{3}\right), \\
f-f_{0}= & \varepsilon F(z) e^{i \omega t}+\text { c.c. }+\varepsilon^{2}\left[F_{2}(z) e^{2 i \omega t}+\right.\text { c.c. } \\
& \left.+f^{m}(z)\right]+O\left(\varepsilon^{3}\right), \\
\widehat{\gamma}-\widehat{\gamma}_{0}= & \varepsilon \widehat{\Gamma}(z) e^{i \omega t}+\text { c.c. }+\varepsilon^{2}\left[\widehat{\gamma}_{2}(z) e^{2 i \omega t}+\right.\text { c.c. } \\
& \left.+\widehat{\gamma}^{m}(z)\right]+O\left(\varepsilon^{3}\right),
\end{aligned}
$$

where $\varepsilon$ is small. The leading-order terms collect the "exact" first harmonic of the periodic oscillation, which can be computed from the exact solution as

$$
\varepsilon(U(r, z), W(r, z))=\frac{1}{T} \int_{0}^{T}(u(r, z, t), w(r, z, t)) e^{-i \omega t} d t,
$$




$$
\varepsilon(F(z), \widehat{\Gamma}(z))=\frac{1}{T} \int_{0}^{T}(f(z, t), \widehat{\gamma}(z, t)) e^{-i \omega t} d t .
$$

In addition to the second harmonic, the second-order terms in Eqs. (15)-(17) comprise the mean values of the velocity field, $\varepsilon^{2}\left(u^{m}(r, z), w^{m}(r, z)\right)$, the free surface deflection, $\varepsilon^{2} f^{m}(z)$, and the surfactant concentration $\varepsilon^{2} \widehat{\gamma}^{m}(z)$. These mean values are calculated in terms of the exact fields as

$$
\begin{gathered}
\left(u^{E}, w^{E}\right) \equiv \varepsilon^{2}\left(u^{m}(r, z), w^{m}(r, z)\right) \\
=\frac{1}{T} \int_{0}^{T}(u(r, z, t), w(r, z, t)) d t, \\
\left(f^{E}, \widehat{\gamma}^{E}\right) \equiv \varepsilon^{2}\left(f^{m}(z), \widehat{\gamma}^{m}(z)\right) \\
=\frac{1}{T} \int_{0}^{T}\left(f(z, t)-f_{0}(z), \widehat{\gamma}(z, t)-\widehat{\gamma}_{0}(z)\right) d t .
\end{gathered}
$$

As can be seen, computing these values requires an accuracy $O\left(\varepsilon^{3}\right)$ in the numerical solver.

Equation (20) defines the components $\left(u^{E}, w^{E}\right)$ of the mean Eulerian velocity. However, the effective mass transport is not given by that quantity, but by the mean Lagrangian velocity defined as the time-averaged velocity along the trajectories of the fluid elements, namely,

$$
\left(u^{L}\left(r_{0}, z_{0}\right), w^{L}\left(r_{0}, z_{0}\right)\right) \equiv \frac{1}{T} \int_{0}^{T}\left(r^{\prime}(t), z^{\prime}(t)\right) d t .
$$

Here, $\left(r_{0}, z_{0}\right)$ is the point where the mean Lagrangian velocity is calculated, and the primes denote the time derivatives. The trajectory $(r(t), z(t))$ of the fluid element passing through $\left(r_{0}, z_{0}\right)$ is calculated by integrating the kinematic equations for the velocity field, $(u, w)=(u(r, z, t), w(r, z, t))$, as

$$
\begin{aligned}
r^{\prime}(t) & =u(r(t), z(t), t), \quad z^{\prime}(t)=w(r(t), z(t), t), \quad \text { with } \\
r(0) & =r_{0} \text { and } z(0)=z_{0} .
\end{aligned}
$$

Equation (22) shows that the exact value of the mean Lagrangian velocity is

$$
\left(u^{L}\left(r_{0}, z_{0}\right), w^{L}\left(r_{0}, z_{0}\right)\right)=\frac{\left(r(T)-r_{0}, z(T)-z_{0}\right)}{T} .
$$

However, and as explained below, this is not a good equation to obtain that quantity. Instead, we may perform a classical asymptotic analysis (as the intensity of the oscillatory flow goes to zero) considering Eq. (22) and the Taylor expansions of the right-hand sides of the kinematic Eqs. (23), namely,

$$
\begin{aligned}
r^{\prime}(t)= & u\left(r_{0}, z_{0}, t\right)+u_{r}\left(r_{0}, z_{0}, t\right)\left[r(t)-r_{0}\right] \\
& +u_{z}\left(r_{0}, z_{0}, t\right)\left[z(t)-z_{0}\right]+\cdots, \\
z^{\prime}(t)= & w\left(r_{0}, z_{0}, t\right)+w_{r}\left(r_{0}, z_{0}, t\right)\left[r(t)-r_{0}\right] \\
& +w_{z}\left(r_{0}, z_{0}, t\right)\left[z(t)-z_{0}\right]+\cdots
\end{aligned}
$$

Substituting Eq. (15) into Eqs. (25) and (26), replacing the resulting equations into Eq. (23), and integrating the result gives

$$
\begin{aligned}
& r(t)-r_{0}=\varepsilon U\left(r_{0}, z_{0}\right) \frac{e^{i \omega t}-1}{i \omega}+\text { c.c. }+O\left(\varepsilon^{2}\right), \\
& z(t)-z_{0}=\varepsilon W\left(r_{0}, z_{0}\right) \frac{e^{i \omega t}-1}{i \omega}+\text { c.c. }+O\left(\varepsilon^{2}\right),
\end{aligned}
$$

where only the leading order terms have been retained. These equations show that $|r(T)-r(0)| \sim|z(T)-z(0)| \sim \varepsilon^{2}$. In other words, calculating the "exact" mean Lagrangian velocity via Eq. (24) is a difficult task because it requires computations that are exact to this order (errors small compared to $\varepsilon^{2}$ ). Instead, we may complete the asymptotic calculation by substituting Eqs. (27), (28), and (15) into Eqs. (25) and (26), and the resulting equation into Eq. (22), which yields

$$
\begin{aligned}
\left(u^{L}, w^{L}\right)= & \varepsilon^{2}\left(u^{m}, w^{m}\right)+\varepsilon^{2}\left[\frac { 1 } { i \omega } \left(\bar{U}_{r} U+\bar{U}_{z} W, \bar{W}_{r} U\right.\right. \\
& \left.\left.+\bar{W}_{z} W\right)+ \text { c.c. }\right]+O\left(\varepsilon^{3}\right) .
\end{aligned}
$$

Hereinafter, the overbar stands for the complex conjugate. In addition, we have taken into account that

$$
\int_{0}^{T} e^{m \omega t} d t=0 \quad \text { if } m= \pm 1, \pm 2, \ldots
$$

Equation (29) is a classical result: the mean Lagrangian velocity equals the mean Eulerian velocity plus the Stokes drift [47], i.e.,

$$
\left(u^{\mathrm{L}}, w^{\mathrm{L}}\right)=\left(u^{\mathrm{E}}, w^{\mathrm{E}}\right)+\left(u^{\mathrm{SD}}, w^{\mathrm{SD}}\right),
$$

where the Stokes drift velocity components are given by

$$
\left(u^{\mathrm{SD}}, w^{\mathrm{SD}}\right) \equiv \frac{\varepsilon^{2}}{i \omega}\left(\bar{U}_{r} U+\bar{U}_{z} W, \bar{W}_{r} U+\bar{W}_{z} W\right)+\text { c.c. }
$$

In contrast to most works mentioned above, the formulas derived here does not rely on the nearly inviscid approximation, but only on the quasilinear approximation Eqs. (15)-(17), which only requires that the oscillatory field be small. In this approximation, the mean Lagrangian velocity field is given to the leading order by Eq. (29) with $(U, W)$ and $\left(u^{m}, w^{m}\right)$ calculated in terms of the (numerically computed) unsteady velocity components $(u, w)$ from Eqs. (18) and (20), respectively. Alternatively, the mean Lagrangian velocity can be calculated using Eq. (24), which is much more computationally demanding. This calculation will be used for the validation of the asymptotic formulas. Similarly, the mean values of the free surface deflection and the surfactant concentration along the free surface are given by Eq. (21).

Using the formulas derived above in the computational domain is somewhat tricky. Note that the calculation of the mean Langrangian velocity must be performed within the physical domain $0 \leqslant r \leqslant f_{0}+f^{E}$, which corresponds to the time-averaged liquid bridge shape. In addition, such calculation must be done for fixed $r$ and $z$, which requires the time-dependent interpolation from the computational domain to the physical one. On the other hand, the computation of the fluid element trajectory used in Eq. (24) requires a double time-dependent interpolation: one to calculate the velocity field at $(r(t), z(t))$ from the velocities at the nodes of the fixed 

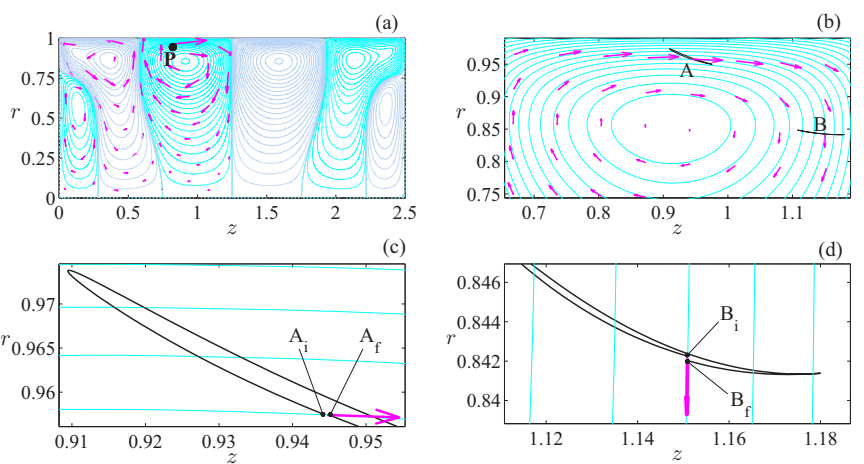

FIG. 3. (a) Streamlines of the mean Lagrangian velocity field. The cyan lines (first, third, and fifth vortex starting from the left) and gray lines (second, fourth, and sixth vortex starting from the left) indicate clockwise and anticlockwise rotations, respectively. The arrows are the mean Lagrangian velocities calculated from Eq. (24). (b) Magnification of plot (a) near the point $(r=0.85, z=0.9)$. The black lines indicate the time-dependent trajectories of the fluid particles A and B calculated from the integration of Eqs. (23). (c and d) Magnification of plot (b) to show the trajectories of the fluid particles $\mathrm{A}$ and $\mathrm{B}$. The subindexes $i$ and $f$ indicate the initial and final positions of the fluid particles in a cycle, respectively. The maximum value of the velocity field is $v_{\max }=1.77 \times 10^{-4}$. The velocity magnitude at the points $\mathrm{P}, \mathrm{A}$, and $\mathrm{B}$ are $0.92 v_{\max }, 0.98 v_{\max }$, and $0.43 v_{\max }$, respectively. The values of the governing parameters are the same as those in Fig. 2, namely, $\Lambda=1.25, V=1, C=0.01$, $\widehat{\gamma_{0}}=0.5, \mathrm{Ma}=0, \omega=2.1$, and $\hat{B}=0.005$.

computational mesh, and another to integrate the kinematic equations in the $(r, z)$ plane. These details are described in the Appendix.

To illustrate the formulas derived above, we calculated the streaming flow for $C=0.01$, Ma $=0, \omega=2.1$, and $\hat{B}=0.005$. We considered a moderately small viscosity, and ignored the Marangoni stress for simplicity. In addition, we selected a representative value of the forcing frequency $\omega$ and amplitude $\hat{B}$. Figure 2 shows the time evolution of the axial component of both the actual time-dependent velocity numerically calculated and the mean Lagrangian velocity. These quantities are evaluated at a representative point next to the mean free surface. As can be seen, the time-dependent velocity reaches a strictly periodic state after the transient stage $0 \leqslant t \lesssim 100$, while the mean Lagrangian velocity becomes stationary for $t \gtrsim 50$. Consistently with the quasilinear assumption implicit in Eq. (15), $w \sim \varepsilon \sim$ 0.05 is small, and $w^{L} \sim \varepsilon^{2}$ is much smaller than $w$ in the periodic regime. Interestingly, the Lagrangian flow reaches its asymptotic value somewhat before the time-dependent velocity becomes periodic, which implies that the mean flow is not a slave of the surface waves. This seemingly means that the streaming flow does affect the dynamics of the primary surface waves, as shown in Refs. [30-34] for small viscosity.

The remaining calculations in this paper will be conducted in the periodic regime arising after the initial transient stage, whose length varies from one case to another. The spatial structure of the streaming flow in the periodic regime is considered in Fig. 3, where a comparison between the "exact"
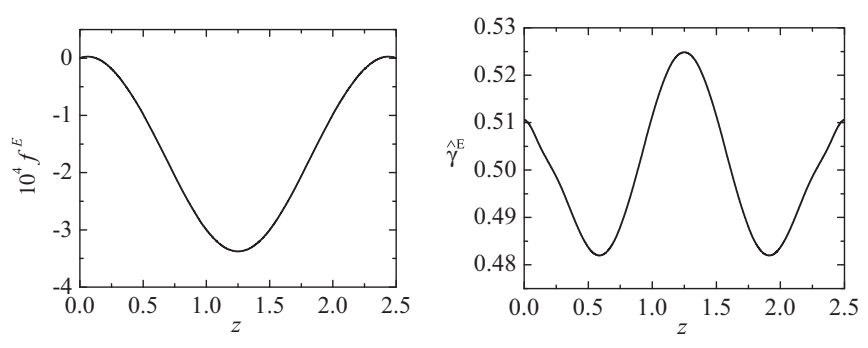

FIG. 4. Mean free surface deflection $f^{E}(z)$ (left) and mean surfactant concentration $\widehat{\gamma}^{E}(z)$ (right). The values of the governing parameters are the same as those in Fig. 2, namely, $\Lambda=1.25, V=1$, $C=0.01, \widehat{\gamma}_{0}=0.5, \mathrm{Ma}=0, \omega=2.1$, and $\hat{B}=0.005$.

and asymptotic formulas [Eqs. (24) and (29), respectively] is made. The results calculated in these two ways are consistent with each other, which constitutes a stringent test for the numerical procedure. It must be noted that nothing similar to these computations has been performed so far, to our knowledge. As can be seen in Fig. 3(a), the streaming flow is reflection-symmetric in about the mean plane $z=1.25$, which is consistent with the invariance of the governing equations under this symmetry. Also, the streamlines show six counter-rotating vortices where the forcing effect of the boundary layers near the disks and the free surface is evident. The free surface boundary layer produces four vortices, which is consistent with the fact that Eq. (29) is quadratic in $U$ and $W$. The first resonant mode of the liquid bridge was excited in this case owing to the selected value of the forcing frequency $\omega$. This mode exhibits two time-dependent vortices associated with the free surface oscillation. As can be seen, quadratic effects double the number of vortices appearing in the streaming flow. The thickness of the boundary layers scales as $\sqrt{C / \omega} \sim 0.07$ in the present case. This value is not small enough for the asymptotic formulas commonly used in the literature to apply. On the other hand, the blow ups in Figs. 3(b)-3(d) illustrate how elongated the fluid trajectories can be, and how small the drift is compared to the widths of those trajectories. These remarks are consistent with the above mentioned difficulties encountered in these computations.

The mean free surface deflection and the mean surfactant concentration for the case analyzed in Fig. 3 are given in Fig. 4. Note that both are reflection symmetric about the mean plane $z=\Lambda$, which is consistent with the invariance of the problem under this symmetry. The left plot indicates that the free surface position is no longer uniform due to the oscillations, although the induced deformation is small. In fact, the mean free surface deflection plays only a secondary role in the context of this paper, and thus it will not be further considered for the sake of brevity. It is interesting to note that $f^{E}(z)<0$ for all $z$, which could be seen as surprising if one takes the conservation of volume into account. However, the liquid bridge volume depends quadratically on $f$ [see Eq. (11)], which means that the time-averaged volume (which must be conserved) does not generally coincide with the volume of the time-averaged free surface location. This well-known artifact produced by nonlinear terms is, in fact, similar to what happens with the time-averaged velocity, which 
is generally nonzero and produces the streaming flow. Note that the volume of the time-averaged free surface location is smaller than its initial value in the present case. The mean surfactant concentration peaks at the liquid bridge center and at the supporting disks and exhibits two minima near $z=\Lambda / 2$ and $3 \Lambda / 2$ (right plot). This behavior is consistent with Fig. 3 , which shows that convection caused by the streaming flow vortices empties the free surface in the regions $z \simeq \Lambda / 2$ and $3 \Lambda / 2$ and fills both the central part of the liquid bridge and the surroundings of the supporting disks.

The simulation illustrated in Figs. 3 and 4 was conducted for $\mathrm{Ma}=0$. Therefore, the surfactant does not alter the surface tension and behaves as a passive scalar in that case. If the scalar were a surface active substance $(\mathrm{Ma}>0)$, then this concentration distribution would cause Marangoni convection in the direction opposite to that of the streaming flow, which would alter significantly both the mean surfactant concentration distribution and the mean flow pattern. This effect will be analyzed in the next section.

\section{RESULTS ON THE INTERACTION OF THE STREAMING FLOW AND MARANGONI CONVECTION}

All the results shown in this section were obtained for the values Eq. (14) of the governing parameters. The strength of the oscillatory and mean flows are measured using RMS values instead of values at particular points, which would be less robust and less appropriate to obtain scaling laws when different configurations are considered. Specifically, the intensity of the oscillatory flow is defined as

$$
I_{\mathrm{osc}}^{\mathrm{bulk}}=\sqrt{\frac{1}{\Lambda V} \int_{0}^{2 \Lambda} \int_{0}^{f^{E}}\left[|U(r, z)|^{2}+|W(r, z)|^{2}\right] r d r d z}
$$

where $U$ and $W$ are given by Eq. (18). The counterpart of Eq. (33) for the streaming flow in the bulk is defined as

$$
I_{\mathrm{mf}}^{\text {bulk }}=\sqrt{\frac{1}{\Lambda V} \int_{0}^{2 \Lambda} \int_{0}^{f^{E}}\left[\left|u^{L}(r, z)\right|^{2}+\left|w^{L}(r, z)\right|^{2}\right] r d r d z}
$$

In addition, the intensity of the mean flow in the free surface is defined as

$$
I_{\mathrm{mf}}^{\mathrm{interf}}=\sqrt{\frac{1}{L_{s}} \int_{0}^{2 \Lambda}\left[\left|u^{L}\right|^{2}+\left|w^{L}\right|^{2}\right]_{r=f^{E}} d z}
$$

where

$$
L_{s}=\int_{0}^{2 \Lambda} \sqrt{1+\left|f_{z}^{E}\right|^{2}} d z
$$

is the length of the time-averaged free surface. Finally, the strength of the mean flow along the axial coordinate is given by

$$
I_{\mathrm{mf}}^{\mathrm{axial}}(z)=\sqrt{2 \int_{0}^{f^{E}(z)}\left[\left|u^{L}(r, z)\right|^{2}+\left|w^{L}(r, z)\right|^{2}\right] r d r .}
$$

The components $u^{L}$ and $w^{L}$ of the mean Lagragian velocities in Eqs. (34), (35), and (37) are given by Eq. (29).
In the following two subsections, we consider the influence on the streaming flow of the two main parameters: the Marangoni number and the strength of the oscillatory flow. As will be seen, the Marangoni number affects the shape of the flow patterns, while the strength of the oscillatory flow influences quantitatively the intensity of the mean flow. We aim at uncovering the main physical effects of Marangoni convection on the streaming flow, rather than a full account of the results obtained as the values of the governing parameters are varied.

\section{A. Influence of the Marangoni number on the flow patterns}

In this section, we consider the dependence of the streaming flow on the Marangoni number for the parameter values listed in Eq. (14). We are mainly interested in illustrating how Marangoni stresses completely change the streaming flow pattern, without intending an exhaustive description of those patterns. For this reason, we will restrict ourselves to the Ohnesorge number $C=0.01$ and the forcing frequency $\omega=2.1$. Smaller values of $C$ would give flow patterns that are more concentrated near the disks and the free surface. The forcing frequency $\omega=2.1$ is close (but not equal) to the first resonance frequency of the liquid bridge for $\mathrm{Ma}=0$, which means that the oscillatory flow intensity is very sensitive to the value of the dynamical Bond number $\hat{B}$. After some calibration, $\hat{B}$ has been chosen such that the maximum over a cycle of $\varepsilon W e^{i \omega t}+$ c.c. at the center of the liquid bridge is 0.05 . This choice makes the intensity of the streaming flow for Ma $=0$ comparable to the strength of the Marangoni convection in the considered range of Ma. It must be noted that the values of $\hat{B}$ resulting from this criterion are physically realistic since they correspond to accelerations that can be easily produced in experiments [48].

Figure 5 shows the counterpart of Fig. 3 for the indicated values of the Marangoni number. As can be seen, all the flow patterns are reflection-symmetric about the mid plane of the liquid bridge. However, and surprisingly at first sight, the streaming flow patterns do not show a monotone behavior as Ma increases. Instead, the six counter-rotating eddies that are present for $\mathrm{Ma}=0$ first merge for $\mathrm{Ma}=0.001,0.005$, and 0.05 , and then give rise to just two counter-rotating eddies for $\mathrm{Ma}=$ 0.1 and 0.2. As Ma increases further, new eddies appear leading to a flow pattern for $\mathrm{Ma}=1$ (very strong Marangoni stress) that is qualitatively similar to that obtained for a much smaller value $\mathrm{Ma}=0.001$. This unexpected behavior is a consequence of the trade off between two mechanisms: nonuniformities in the surfactant concentration at the free surface, and the intensity of the oscillatory flow.

The surfactant concentration along the free surface is shown in Fig. 6 for the same values of the Marangoni number considered in Fig. 5. As can be seen, the surfactant concentration becomes almost spatially uniform for very small Marangoni stress. This is due to the strong stabilizing effect of this stress, and is consistent with our guess at the end of Sec. II B. This behavior explains the streaming flow patterns shown in Fig. 5 for $\mathrm{Ma}=0.001,0.005$, and 0.05. However, as $\mathrm{Ma}$ is increased further, the strength of the oscillatory flow increases, peaks at $\mathrm{Ma} \simeq 0.2$, and decreases again [Fig. 7(a)]. 

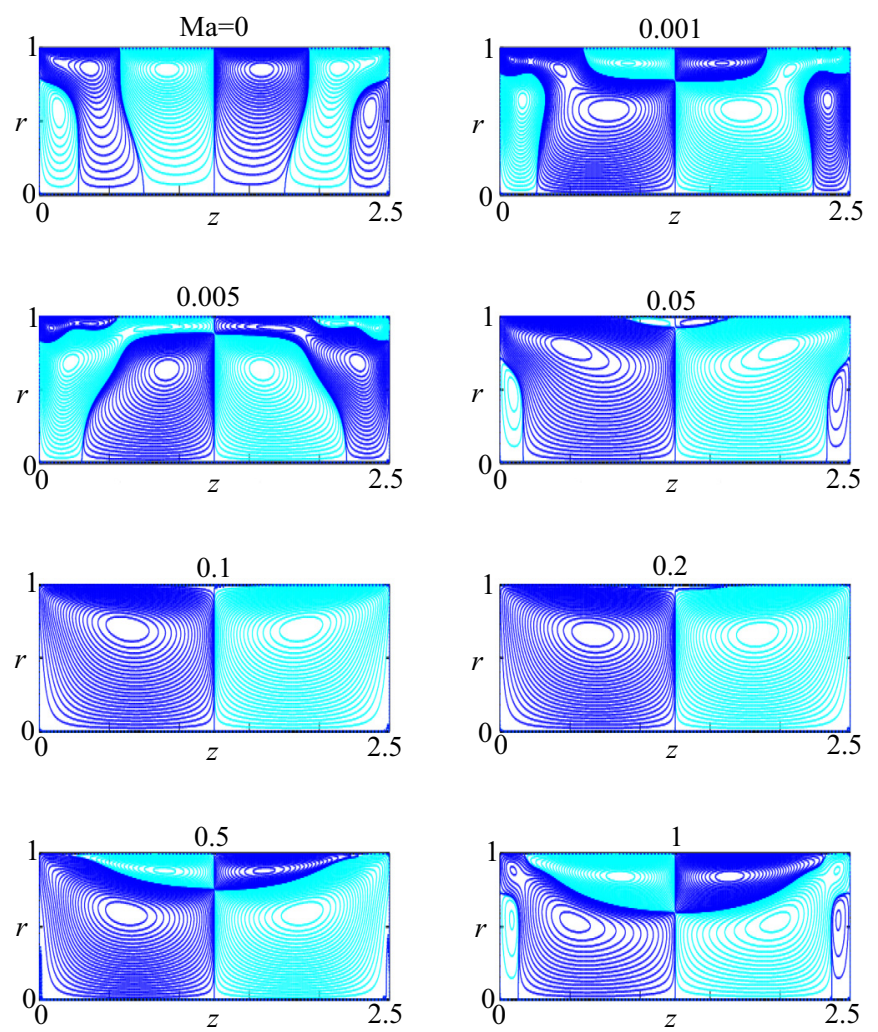

FIG. 5. Counterpart of Fig. 3 for different Marangoni numbers as indicated by the labels in the figure. The values of the governing parameters are $\Lambda=1.25, V=1, C=0.01, \widehat{\gamma}_{0}=0.5$, and $\omega=2.1$.

This is due to the fact that the forcing frequency matches the resonance frequency of the contaminated liquid bridge for $\mathrm{Ma} \simeq 0.2$. Since the intensity of the streaming flow depends on that of the oscillatory flow, they follow similar trends, as the comparison between Fig. 7(a) and Figs. 7(b) and 7(c) shows.

Summarizing the above, the Marangoni stress has a great quantitative and qualitative influence on the streaming flow patterns. As Ma increases, these patterns do not behave monotonously due to the fairly complex behavior of both the surfactant concentration at the free surface and the strength of the nearly resonant oscillation.
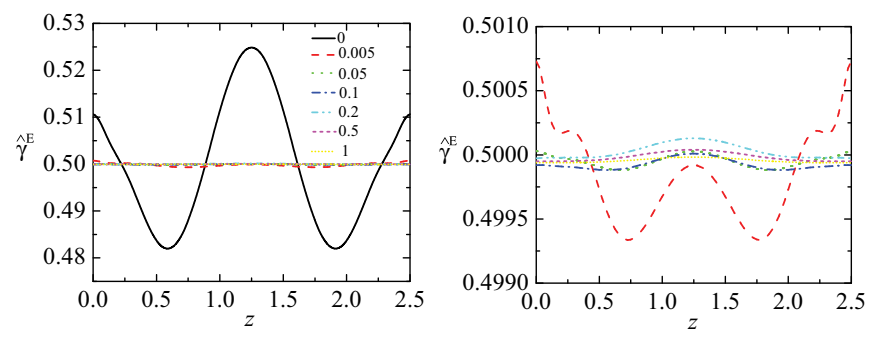

FIG. 6. Mean surfactant concentration $\widehat{\gamma}^{E}(z)$. The colors (and types of lines) correspond to the different Marangoni numbers as indicated in the left-hand graph. The values of the rest of governing parameters are $\Lambda=1.25, V=1, C=0.01, \widehat{\gamma}_{0}=0.5$, and $\omega=2.1$.

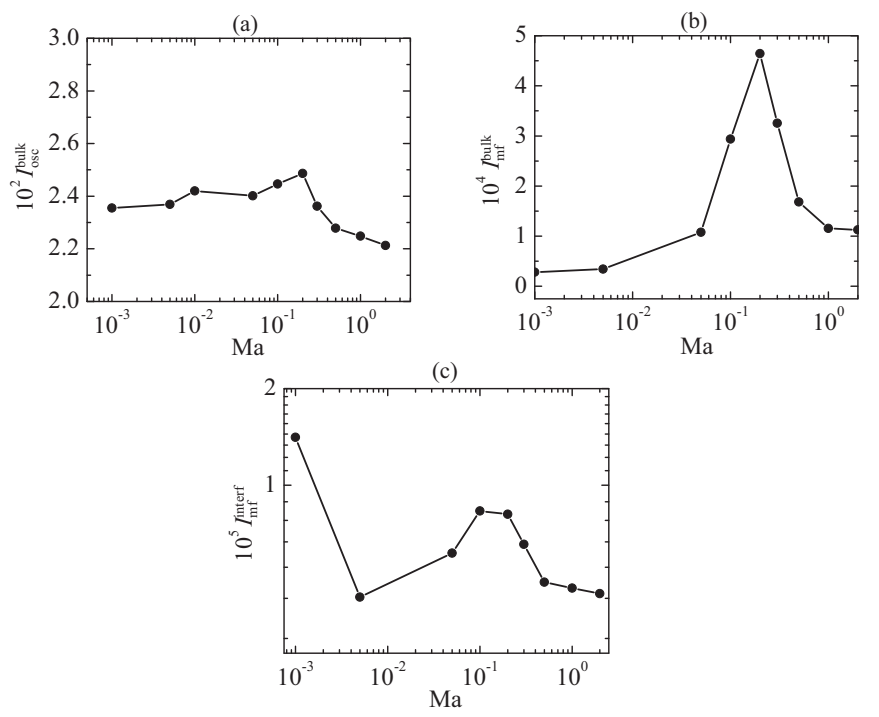

FIG. 7. Intensities $I_{\mathrm{osc}}^{\text {bulk }}$ (a), $I_{\mathrm{mf}}^{\text {bulk }}$ (b), and $I_{\mathrm{mf}}^{\text {interf }}$ (c) of the oscillatory flow, the mean flow in the bulk and the mean flow in the interface, respectively, for the Marangoni numbers considered in Fig. 5. The values of the rest of governing parameters are $\Lambda=1.25$, $V=1, C=0.01, \widehat{\gamma}_{0}=0.5$, and $\omega=2.1$.

\section{B. Influence of the oscillatory flow intensity}

In this section, we analyze the influence of the oscillatory flow intensity on the streaming flow intensity for the parameter values listed in Eq. (14). This analysis is more involved than that presented in the previous section due to the larger number of parameters considered, and the fact that hysteresis could be present because viscous effects are small and the forcing frequency is close to the resonant one. The results are obtained by varying the dynamical Bond number. However, and to uncover the underlying scaling laws for the intensity of streaming flow in the bulk and the interface, these quantities are plotted in terms of the oscillatory flow intensity.

Figure 8 shows the influence of $I_{\mathrm{osc}}^{\text {bulk }}$ on $I_{\mathrm{mf}}^{\text {bulk }}$ and $I_{\mathrm{mf}}^{\mathrm{interf}}$ for the indicated Marangoni numbers, $C=0.01$ and $\omega=2.1$. As can be seen, $I_{\mathrm{mf}}^{\text {bulk }}$ scales as $\left(I_{\mathrm{osc}}^{\text {bulk }}\right)^{2}$ for all values of Ma. This quadratic scaling law can be expected in view of Eq. (29). The curves $I_{\mathrm{mf}}^{\text {bulk }}$ versus $I_{\mathrm{osc}}^{\text {bulk }}$ are parallel to each other. The maximum and minimum values of the scaling factor differ by one order of magnitude. This factor does not behave monotonously as Ma increases, but first decreases and then
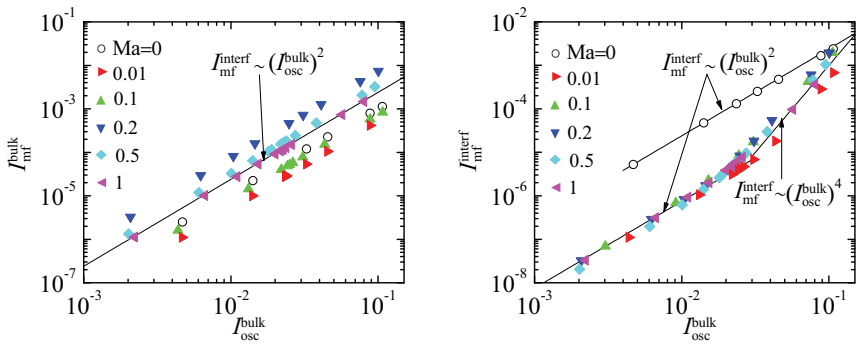

FIG. 8. $I_{\mathrm{mf}}^{\text {bulk }}$ (left) and $I_{\mathrm{mf}}^{\mathrm{interf}}$ (right) vs. $I_{\mathrm{osc}}^{\text {bulk }}$ for the indicated Marangoni numbers. The values of the rest of governing parameters are $\Lambda=1.25, V=1, C=0.01, \widehat{\gamma}_{0}=0.5$, and $\omega=2.1$. 

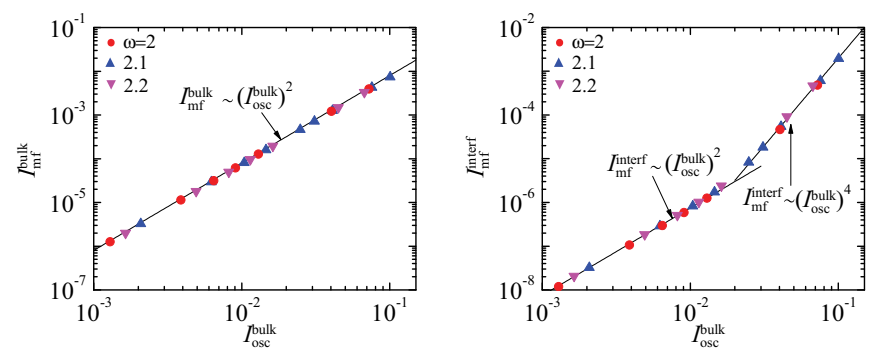

FIG. 9. Counterpart of Fig. 8 for the indicated oscillation frequencies. The values of the rest of governing parameters are $\Lambda=1.25$, $V=1, C=0.01, \widehat{\gamma_{0}}=0.5$, and $\mathrm{Ma}=0.2$.

increases. This is consistent with the non-monotone behavior of the streaming flow patterns as Ma increases (Fig. 5). The curves $I_{\mathrm{mf}}^{\text {interf }}$ versus $I_{\mathrm{osc}}^{\text {bulk }}$ clearly show two power-law regimes: one for small $I_{\mathrm{osc}}^{\text {bulk }}$ and another for large $I_{\mathrm{osc}}^{\text {bulk}}$, with a fairly well defined threshold at $I_{\text {osc }}^{\text {bulk }} \simeq 0.02$. Moreover, the curves for $\mathrm{Ma}>0$ are very close to each other, and clearly deviate from that for $\mathrm{Ma}=0$.

The same behavior is found for other forcing frequencies, as shown in Fig. 9 for $\mathrm{Ma}=0.2$. As in the previous cases, this figure shows a unique scaling law for $I_{\mathrm{mf}}^{\text {bulk }}$ and two scaling laws for $I_{\mathrm{mf}}^{\text {bulk }}$ with a well defined threshold in $I_{\text {osc }}^{\text {bulk }}$. This threshold is very close to that in Fig. 8. In addition, the scaling laws almost coincide for the three values of $\omega$, which suggests that these laws show a fairly weak dependence on the forcing frequency.

The scaling behavior found in Figs. 8 and 9 is also present for smaller values of $C$, as shown in Fig. 10. As can be seen, the scaling laws for $I_{\mathrm{mf}}^{\text {bulk }}$ and $I_{\mathrm{mf}}^{\text {interf }}$ coincide with their counterparts in the previous cases. Moreover, the curve $I_{\mathrm{mf}}^{\text {bulk }}$ versus $I_{\mathrm{osc}}^{\text {bulk }}$ almost coincide for the two values of $C$, in spite of the fact that these are disparate. On the other hand, the curves $I_{\mathrm{mf}}^{\mathrm{interf}}$ versus $I_{\text {osc }}^{\text {bulk }}$ slightly deviate from each other. The transition between the two scaling behaviors is less sharp for the smallest value of $C$.

Summarizing the above, the intensity of the streaming flow in the interface shows two well defined power-law behaviors, one for small oscillatory flow intensity and another for larger values of this quantity. These behaviors are fairly universal, since they show the same exponents and the same transition threshold for the considered values of the Marangoni number, forcing frequency, and Ohnesorge number. Such regimes are reminiscent of those found in Ref. [39] (see Fig. 4 in that paper) for the streaming flow produced by Faraday waves in
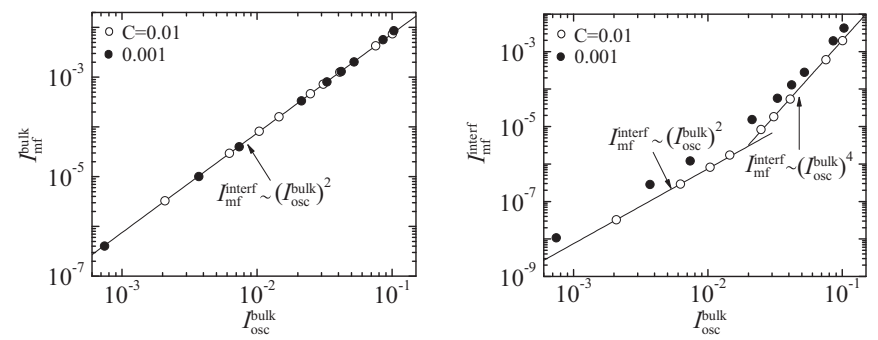

FIG. 10. Counterpart of Fig. 8 for the indicated values of $C$. The values of the rest of governing parameters are $\Lambda=1.25, V=1$, $\widehat{\gamma_{0}}=0.5, \mathrm{Ma}=0.2$, and $\omega=2.1$.

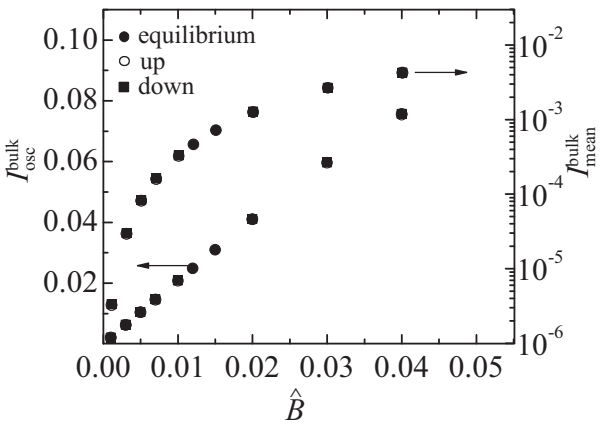

FIG. 11. $I_{\mathrm{osc}}^{\text {bulk }}$ and $I_{\mathrm{mf}}^{\text {bulk }}$ as a function of the dynamical Bond number $\hat{B}$ starting from equilibrium, as well as starting from the previous point while increasing (up) and decreasing (down) progressively the dynamical Bond number. The symbols overlap. The values of the governing parameters are $\Lambda=1.25, V=1, C=0.01$, $\widehat{\gamma}_{0}=0.5, \mathrm{Ma}=0.2$, and $\omega=2.1$.

a rectangular container with tap water. However, we cannot ensure that there is a plain analogy between our problem and those experiments for two reasons. First, the data in Ref. [[39], Fig. 4] were not plotted on log-log scale, meaning that the power law for small oscillatory flow intensity could not be uncovered in that paper. In addition, tap water was most seemingly contaminated with a soluble surfactant in those experiments.

Finally, we aim at elucidating whether hystheresis is present as the dynamic Bond number is varied. As already mentioned, this could occur since viscous effects are weak and the forcing frequency is close to the first resonant frequency. Figure 11 shows the intensities of the oscillatory and mean flows in the bulk obtained when the periodic regime is reached starting from equilibrium, as well as starting from the previous point while increasing or decreasing progressively the dynamical Bond number $\hat{B}$. As can be seen, the liquid bridge oscillations do not not exhibit hystheresis, i.e., they do not depend on the liquid bridge history.

\section{CONCLUSIONS}

We have developed a consistent theory of the interaction between the streaming flow produced by axial vibrations in a liquid bridge and Marangoni convection due to the presence of an insoluble surfactant monolayer. In contrast to previous papers in the field, this theory does not rely on any nearly inviscid formulation, but only on the assumption that the forcing amplitude is sufficiently low for the steepness of the free surface deflection to be small. The streaming flow intensities have been calculated from fully nonlinear direct numerical simulations. Two sets of formulas have been derived and used for cross-checking: one "exact" and based on the average along the trajectories of the fluid elements and another asymptotic and based on the addition of the Eulerian mean velocity and Stokes drift. After validation, the (computationally cheaper) asymptotic formulas have been used in the rest of computations. The main results of this paper were obtained for a realistic set of parameter values. In particular, the forcing frequency was very close to the first resonant frequency of the liquid bridge. 
The following general conclusions can be established:

(i) The streaming flow pattern is highly affected by Marangoni stress even for very small values of Ma. Moreover, the flow patterns show a nonmonotonous behavior as $\mathrm{Ma}$ is increased, which is seemingly due to two main effects: the tendency of Marangoni stresses to oppose any surfactant inhomogeneities in the free surface and the nonmonotonous oscillatory response of the liquid bridge due to resonance peaks whose frequencies depend on Ma.

(ii) The strength of the streaming flow shows a "universal" behavior with a unique power law for the intensity in the bulk and a transition between two power laws for the intensity in the free surface. This could be seen as somewhat similar to some recent findings in the literature [39].

The above conclusions are expected to apply to other vibrating systems despite the peculiarities of the primary oscillating flow producing the mean flow. In fact, we have selected the liquid bridge geometry only because it shows fairly simple oscillatory flow patterns when the first resonant mode is excited. This allowed us to focus on the main involved physical mechanisms.

The scaling laws obtained in the present analysis require that the forcing amplitude be small. For larger amplitudes, the dynamics are fully nonlinear and much more complex. In this case, the oscillatory and mean flow components of the state variables are not expected to be related by simple laws. On the other hand, the somewhat small viscous effects considered in this paper might also play a role. Although the quadratic dependence of the mean flow on the vibrating amplitude also holds for more viscous fluids, the interaction with Marangoni convection may change that dependence for not too small vibrating amplitudes. The analysis of this possibility is beyond the scope of this paper, where only realistic values of the parameters have been considered.

Most of surface-active substances in nature are watersoluble. Surfactant solubility makes our analysis less general and does affect the results of the paper quantitatively (in particular, the scaling laws). However, there is a large set of surfactants characterized by adsorption and desorption time scales much larger than the liquid bridge capillary time. The results presented here can be extended to such substances. In any case, the fact that Marangoni elasticity opposes surfactant inhomogeneities produced by the streaming flow remains valid for soluble surfactants as well.

\section{ACKNOWLEDGMENTS}

This research has been partially supported by the Spanish Ministry of Economy, Industry and Competitiveness under Grants No. TRA2016-75075-R and No. DPI2016-78887-R.

\section{APPENDIX: COMPUTATIONS OF THE LAGRANGIAN MEAN VELOCITIES IN THE NUMERICAL DOMAIN}

As explained in Sec. II A, the computational spatial coordinates are $\eta \equiv r / f(z, t)$ and $z$. The counterparts of Eqs. (15),
(18), and (20) for the velocity components are

$$
\begin{aligned}
& (\widetilde{u}, \widetilde{w})=\varepsilon\left[(\tilde{U}(\eta, z), \widetilde{W}(\eta, z)) e^{i \omega t}+\text { c.c. }\right] \\
& +\varepsilon^{2}\left[\left(\widetilde{U}_{2}(\eta, z), \widetilde{W}_{2}(\eta, z)\right) e^{2 i \omega t}\right. \\
& \left.+ \text { c.c. }+\left(\widetilde{u}^{m}(\eta, z), \widetilde{w}^{m}(\eta, z)\right)\right]+O\left(\varepsilon^{3}\right), \\
& \varepsilon(\widetilde{U}(\eta, z), \widetilde{W}(\eta, z))=\frac{1}{T} \int_{0}^{T}(\widetilde{u}(\eta, z, t), \widetilde{w}(\eta, z, t)) e^{-i \omega t} d t, \\
& \varepsilon^{2}\left(\widetilde{u}^{m}(\eta, z), \widetilde{w}^{m}(\eta, z)\right)=\frac{1}{T} \int_{0}^{T}(\widetilde{u}(\eta, z, t), \widetilde{w}(\eta, z, t)) d t,
\end{aligned}
$$

where the tilde indicates that the quantity is evaluated in the fixed numerical domain. In addition, Eq. (16) implies that

$$
\eta=\frac{r}{f}=\frac{r}{f_{0}}-\varepsilon\left[\frac{r F}{f_{0}^{2}} e^{i \omega t}+\text { c.c. }\right]+O\left(\varepsilon^{2}\right) .
$$

The Eulearian mean velocity at a fixed point $(r, z)$ can be calculated from the velocity field in the numerical domain as

$\left(u^{E}(r, z), w^{E}(r, z)\right)=\frac{1}{T} \int_{0}^{T}[\tilde{u}(r / f(t), z, t), \widetilde{w}(r / f(t), z, t)] d t$.

This calculation involves the evaluation of the velocity field at a moving point $(r / f(t), z)$ of the numerical domain in the course of the oscillation. To avoid this evaluation, the above integral is expanded in the following way

$$
\begin{aligned}
\frac{1}{T} \int_{0}^{T}[\tilde{u}(r / f(t), z, t), \tilde{w}(r / f(t), z, t)] d t \\
=\frac{1}{T} \int_{0}^{T}\left(\widetilde{u}\left(r / f_{0}, z, t\right), \tilde{w}\left(r / f_{0}, z, t\right)\right) d t \\
\quad-\varepsilon^{2}\left[\frac{r \bar{F}(z)}{f_{0}^{2}}\left(U_{\eta}\left(r / f_{0}, z\right), W_{\eta}\left(r / f_{0}, z\right)\right)+\text { c.c. }\right]+O\left(\varepsilon^{3}\right) .
\end{aligned}
$$

Therefore, the Eulearian mean velocity at a fixed point $(r, z)$ can be obtained as

$$
\begin{aligned}
\left(u^{E}, w^{E}\right)= & \varepsilon^{2}\left[\left(\tilde{u}^{m}\left(\eta_{0}, z\right), \widetilde{w}^{m}\left(\eta_{0}, z\right)\right)\right. \\
& \left.-\frac{\eta_{0} \bar{F}(z)}{f_{0}(z)}\left(\widetilde{U}_{\eta}\left(\eta_{0}, z\right), \widetilde{W}_{\eta}\left(\eta_{0}, z\right)\right)+\text { c.c. }\right]+O\left(\varepsilon^{3}\right),
\end{aligned}
$$

where $\eta_{0} \equiv r / f_{0}(z)$ is a fixed coordinate in the numerical domain. To leading order, $\widetilde{U}(r / f, z)=\widetilde{U}\left(r / f_{0}, z\right)$ and $\widetilde{W}(r / f, z)=\widetilde{W}\left(r / f_{0}, z\right)$. Therefore, the Lagrangian mean velocity at a fixed point $(r, z)$ can be calculated as

$$
\begin{aligned}
\left(u^{L}, w^{L}\right)= & \varepsilon^{2}\left(\widetilde{u}^{m}, \widetilde{w}^{m}\right) \\
& +\varepsilon^{2}\left[-\frac{\eta_{0} \bar{F}}{f_{0}}\left(\widetilde{U}_{\eta}, \widetilde{W}_{\eta}\right)+\frac{1}{i \omega}\left(\widetilde{U}_{\eta} \widetilde{U}+\widetilde{U}_{z} \widetilde{W}, \widetilde{W}_{\eta} \widetilde{U}\right.\right. \\
& \left.\left.+\overline{\widetilde{W}}_{z} \widetilde{W}\right)+ \text { c.c. }\right]+O\left(\varepsilon^{3}\right),
\end{aligned}
$$


where all the functions are evaluated at $\left(\eta_{0}, z\right)$. Finally, the kinematic Eqs. (23) are rewritten as

$$
\frac{d \eta}{d t}=\frac{\widetilde{u}(\eta, z, t)}{f(z, t)}-\frac{\eta f_{z}(z, t) \tilde{w}(\eta, z, t)}{f(z, t)}-\frac{\eta f_{t}(z, t)}{f(z, t)}, \quad \frac{d z}{d t}=\widetilde{w}(\eta, z, t) .
$$

[1] J. W. S. Lord Rayleigh, On the circulation of air observed in Kundt's tubes, and on some allied acoustical problems, Philos. Trans. R. Soc. London 175, 1 (1884).

[2] M. Faraday, On the forms and states assumed by fluids in contact with vibrating elastic surfaces, Philos. Trans. R. Soc. London A 121, 319 (1831).

[3] J. W. S. Lord Rayleigh, Theory of Sound, Vol. I (Dover, New York, 1945).

[4] S. Taylor, Colours shown by thin liquid films under the action of sonorous vibrations, Proc. R. Soc. Lond. 27, 71 (1878).

[5] J. M. Vega, F. J. Higuera, and P. D. Weidman, Quasi-steady vortical structures in vertically vibrating soap films, J. Fluid Mech. 372, 213 (1998).

[6] H. Schlichting, Berechnung ebener periodischer Grenzschichtströmungen, Phys. Z 33, 327 (1932).

[7] H. Schlichting, Boundary Layer Theory (McGraw-Hill, New York, 1968).

[8] M. S. Longuet-Higgins, Mass transport in water waves, Philos. Trans. R. Soc. A 245, 535 (1953).

[9] G. K. Batchelor, An Introduction to Fluid Dynamics (Cambridge University Press, Cambridge, 1967).

[10] J. Lighthill, Acoustic streaming, J. Sound Vib. 61, 391 (1978).

[11] N. Riley, Steady streaming, Annu. Rev. Fluid Mech. 33, 43 (2001).

[12] N. Padmanabhan and T. J. Pedley, Three-dimensional steady streaming in a uniform tube with an oscillating elliptical crosssection, J. Fluid Mech. 178, 325 (1987).

[13] J. Lighthill, Acoustic streaming in the ear itself, J. Fluid Mech. 239, 551 (1992).

[14] N. Riley, Acoustic streaming about a cylinder in orthogonal beams, J. Fluid Mech. 242, 387 (1992).

[15] B. Yan, D. B. Ingham, and B. R. Morton, Streaming flow induced by an oscillating cascade of circular cylinders, J. Fluid Mech. 252, 147 (1993).

[16] B. Moudjed, V. Botton, D. Henry, H. Ben Hadid, and J.-P. Garandet, Scaling and dimensional analysis of acoustic streaming jets, Phys. Fluids 26, 093602 (2014).

[17] M. S. Longuet-Higgins, Viscous streaming from an oscillating spherical bubble, Proc R. Soc. Lond. A 454, 725 (1998).

[18] O. M. Phillips, The Dynamics of the Upper Ocean (Cambridge University Press, Cambridge, 1977).

[19] A. K. Liu and S. H. Davis, Viscous attenuation of mean drift in water waves, J. Fluid Mech. 81, 63 (1977).

[20] A. D. D. Craik, The drift velocity of water waves, J. Fluid Mech. 116, 187 (1982).

[21] A. D. D. Craik, Wave Interactions and Fluid Flows (Cambridge University Press, Cambridge, 1985).

[22] M. Kubota, A mechanism for the accumulation of floating marine debris north of Hawaii, J. Phys. Oceanogr. 24, 1059 (1994).

[23] S. Leibovich, On wave-current interaction theories of Langmuir circulations, Annu. Rev. Fluid Mech. 15, 391 (1983).
[24] T. M. Squires and S. R. Quake, Microfluidics: Fluid physics at the nanoliter scale, Rev. Mod. Phys. 77, 977 (2005).

[25] J. A. Nicolas, D. Rivas, and J. M. Vega, The interaction of thermocapillary convection and low-frequency vibration in nearly inviscid liquid bridges, Z. Angew. Math. Phys. 48, 389 (1997).

[26] D. Lyubimov, T. Lyubimova, and B. Roux, Mechanisms of vibrational control of heat transfer in a liquid bridge, Int. J. Heat Mass Transfer, 40, 4031 (1997).

[27] J. A. Nicolas, D. Rivas, and J. M. Vega, On the steady streaming flow due to high frequency vibration in nearly inviscid liquid bridges, J. Fluid Mech. 354, 147 (1998).

[28] T. Lyubimova, R. V. Scuridin, A. Cröll, and P. Dold, Influence of high frequency vibrations on fluid flow and heat transfer in a floating zone, Cryst. Res. Technol. 38, 635 (2003).

[29] Q. Wan, T. Wu, J. Chastain, W. L. Roberts, A. V. Kuznetsov, and P. I. Ro, Streaming in a narrow channel established by a vibrating piezoelectric bimorph, Flow Turbul. Combust. 74, 195 (2005).

[30] C. Martel, E. Knobloch, and J. M. Vega, Dynamics of counterpropagating waves in parametrically forced systems, Physica D 137, 94 (2000).

[31] M. Higuera, J. A. Nicolas, and J. M. Vega, Weakly nonlinear nonaxisymmetric oscillations of capillary bridges at small viscosity, Phys. Fluids 14, 3251 (2002).

[32] E. Martin, C. Martel, and J. M. Vega, Drift instability of standing Faraday waves, J. Fluid Mech. 467, 57 (2002).

[33] M. Higuera, J. M. Vega, and E. Knobloch, Coupled amplitudestreaming ow equations for nearly inviscid Faraday waves in small aspect ratio containers, J. Nonlinear Sci. 12, 505 (2002).

[34] M. Higuera, E. Knobloch, and J. M. Vega, Dynamics of nearly inviscid Faraday waves in almost circular containers, Physica D 201, 83 (2005).

[35] M. C. Cross and P. C. Hohenberg, Dynamics of nearly inviscid Faraday waves in almost circular containers, Rev. Mod. Phys. 65, 851 (1993).

[36] E. Martin and J. M. Vega, The effect of surface contamination on the drift instability of standing Faraday waves, J. Fluid Mech. 546, 203 (2006).

[37] S. Douady, S. Fauve, and O. Thual, Oscillatory phase modulation of parametrically forced surface waves, Europhys. Lett. 10, 309 (1989).

[38] S. L. Strickland, M. Shearer, and K. E. Daniels, Spatiotemporal measurement of surfactant distribution on gravity-capillary waves, J. Fluid Mech. 777, 523 (2015).

[39] N. Périnet, P. Gutiérrez, H. Urra, N. Mujica, and L. Gordillo, Streaming patterns in Faraday waves, J. Fluid Mech. 819, 285 (2017).

[40] H. Punzmann, N. Francois, H. Xia, G. Falkovich, and M. Shats, Generation and reversal of surface flows by propagating waves, Nat. Phys. 10, 658 (2014). 
[41] M. A. Herrada, J. M. Montanero, and J. M. Vega, The effect of surface shear viscosity on the damping of oscillations in millimetric liquid bridges, Phys. Fluids 23, 082102 (2011).

[42] A. Ponce-Torres, M. A. Herrada, J. M. Montanero, and J. M. Vega, Linear and nonlinear dynamics of an insoluble surfactant-laden liquid bridge, Phys. Fluids 28, 112103 (2016).

[43] J. A. Nicolas and J. M. Vega, Weakly nonlinear oscillations of nearly-inviscid axisymmetric liquid bridges, J. Fluid Mech. 328, 95 (1996).

[44] D. Lyubimov, T. Lyubimova, R. V. Skuridin, G. Chen, and B. Roux, Numerical investigation of meniscus deformation and flow in an isothermal liquid bridge subject to high-frequency vibrations under zero gravity conditions, Comput. Fluids 31, 663 (2002).

[45] Y.-M. Tricot, Surfactants: Static and Dynamic Surface Tension (Chapman and Hall, London, 1997), pp. 100-136

[46] M. A. Herrada and J. M. Montanero, A numerical method to study the dynamics of capillary fluid systems, J. Comput. Phys. 306, 137 (2016).

[47] G. G. Stokes, On the theory of oscillatory waves, Trans. Cambridge Phil. Soc. 8, 441 (1847).

[48] C. Ferrera, M. G. Cabezas, and J. M. Montanero, An experimental analysis of the linear vibration of axisymmetric liquid bridges, Phys. Fluids 18, 082105 (2006). 